\title{
PARTICULARITÉS BIOLOGIQUES DE LA MÉTACERCAIRE DE POSTHODIPLOSTOMUM CUTICOLA (VON NORDMANN)
}

\author{
Par J. CALLOT
}

Posthodiplostomum cuticola (von Nordmann 1832) Dubois 1936 a déjà fait l'objet de nombreuses et intéressantes recherches de la part de Ciurea. Cet auteur a étudié, en particulier, l'évolution de la métacercaire et des formes jeunes par infestation de différents hôtes. Il a aussi montré que le distome étudié par Scheuring et Eversbuch, comme la forme jeune de Strigea cornu, était en réalité P. cuticola.

La métacercaire de ce trématode, connue depuis longtemps sous le nom de Distomum cuticola, infeste les cyprinidés des environs de Strasbourg, en particulier dans les bras morts du Rhin. En certains points, situés au voisinage de héronnières, 80 à $90 \mathrm{p}, 100$ des poissons blancs sont porteurs des lésions caractéristiques dues à ce ver.

J'ai entrepris un certain nombre d'expériences sur la vitalité de cette métacercaire placée dans.différentes conditions.

\section{Métacercaire NON ISOLÉE de L'Hôte}

A. Action du froid. - La métacercaire, lorsque le poisson qui la contient est conservé à une température inférieure à zéro, meurt extrêmement rapidement (contrairement à celle de $O$. felineus ou de $H$. heterophyes).

B. Putréfaction. - Par contre, la métacercaire résiste au moins trois jours dans son hôte en pleine putréfaction. Dans l'eau, cette résistance nous a paru encore plus grande.

C. Eau salée. - Dans l'eau salée physiologique, les fragments de poisson, prélevés sans précautions d'asepsie, entrent en putréfaction, mais la métacercaire résiste de quatre à cinq jours.

J'ai essayé des saumures variées. Dans une eau contenant 25 gr. de chlorure de sodium par litre, les métacercaires ont été retrouvées vivantes après neuf jours.

Annales de Parasitologie, T. XVII, ${ }^{\circ} 4 .-1^{\text {er }}$ juillet 1939, p. 332-335. 


\section{MÉtacercaires enkystées isolées}

Des kystes, isolés le plus complètement possible du muscle de poisson et des formations réactionnelles, placés dans de l'eau salée physiologique stérile, ont été conservés à la température du laboratoire pendant 67 jours. Au bout de ce temps, les métacercaires étaient parfaitement vivantes, mobiles dans le kyste transparent, comme au premier jour. Elles n'avaient pas évacué le contenu de leur appareil excréteur.

Milieux de culture. - Des métacercaires, entourées encore d'une légère couche de tissu réactionnel (impossible à enlever complètement, si l'on veut opérer aseptiquement, sans traumatiser le kyste hyalin), ont été placées dans deux types de milieux. Le premier était constitué par du plasma de carpe et du suc musculaire de gardon, le second par du plasma de canard et du muscle de gardon.

Dans le premier milieu, les métacercaires ont parfaitement vécu pendant 44 jours à la température du laboratoire.

Dans le second milieu, la métacercaire a vécu 60 jours, cependant que les fragments de tissu de poisson qui adhéraient au kyste parasitaire donnaient des proliférations remarquables. Cette expérience, faite à la température du laboratoire, dût être interrompue à la suite de l'envahissement du milieu par un champignon.

Greffe. - Des métacercaires, prélevées dans les mêmes conditions que les précédentes, ont été greffées à différents poissons (carpes, carassius, gardons, perches) et à des axolotls.

Les kystes ainsi greffés dans le muscle ou le tissu sous-dermique ont survécu un temps plus ou moins long. C'est ainsi que, chez une perche jeune (hôte naturel rare), le kyste, greffé le 13 janvier dans les muscles dorsaux, vivait encore le 8 mars, lorsqu'il fut retrouvé chez la perche morte ce jour-là. Ce kyste fut placé dans de l'eau physiologique salée et vivait encore le 10 avril, soit 86 jours après l'extirpation primitive.

Dans un kyste greffé chez une carpe, la métacercaire vivait encore 22 jours après l'opération. Ce kyste fut ensuite passé dans l'eau salée physiologique pendant quelques jours, puis dans l'eau glycosée isotonique et enfin fixé 38 jours après sa première extraction.

Par contre, chez l'axolotl et chez les gardons, les kystes furent détruits peu après la greffe, soit pour des raisons physiologiques (axolotl), soit par suite de traumatismes au cours de l'opération. Il paraissait logique d'escompter une longue survie chez le gardon, hôte primitif des métacercaires employées. 
Les métacercaires extraites des kystes après ces différentes opérations, effectuées à la température du laboratoire, ne présentaient aucune trace d'évolution.

Digestion artificielle. - Je me suis servi d'un liquide contenant :

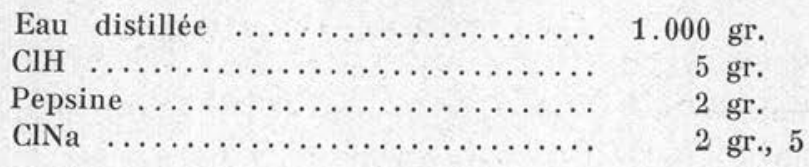

A la température du laboratoire, des fragments de poissons infestés étaient placés dans des verres coniques contenant ce liquide. Au bout de quelques heures, je pouvais récolter des kystes réduits à l'enveloppe hyaline et contenant des métacercaires bien vivantes.

Lorsque cette opération avait lieu à l'étuve à $37^{\circ}$, les kystes s'ouvraient, sans dommages immédiats pour la métacercaire. A la température du laboratoire, l'action des sels biliaires et de la trypsine fut nulle sur les kystes.

A $37^{\circ}$, les kystes placés dans des dilutions variées de ces sels réagissent irrégulièrement; les uns s'ouvrent, les autres pas ; parfois la métacercaire meurt à l'intérieur du kyste sans qu'il $y$ ait une évolution quelconque.

Température. - J'ai essayé quelle pouvait être, en dehors de la digestion, l'influence de la température sur les kystes ou les métacercaires extraites de kystes.

Des kystes ou des métacercaires, placés dans des liquides alcalins pendant un temps variant de 24 à 48 heures, ne présentèrent pas trace d'évolution et moururent le plus souvent avant la fin de l'expérience.

Cependant, en examinant des métacercaires enkystées ayant séjourné 48 heures à $37^{\circ}$ dans de l'eau salée physiologique, j'ai trouvé qu'une sur trois présentait un développement nettement plus marqué des organes génitaux. Les testicules, au lieu d'être de simples ébauches, avaient l'aspect compact de glandes, l'ovaire était bien net, l'utérus marqué par une bande longitudinale entre les branches du testicule postérieur. La bourse copulatrice enfin était très développée. L'aspect du distome rappelle tout à fait alors celui qui est figuré par Scheuring et Eversbuch (fig. 1, p. 46) et qui représente une forme jeune de l'intestin du héron. Dans le cas de cet exemplaire, il n'y a toutefois pas d'indication de l'utérus.

Il s'agit peut-être, dans le cas que je rapporte, d'un exemplaire plus âgé, plus évolué et qui aurait été trouvé dans cet état chez le 
poisson. Cependant, je n'ai jamais observé un tel degré de développement parmi les très nombreux exemplaires que j'ai extrait directement de poissons.

\section{RÉsumé}

J'indique dans cette note quelques faits biologiques concernant la métacercaire de $P$. cuticola, en particulier la grande résistance de la forme enkystée à la putréfaction, à la salure du milieu ; la vitalité considérable dans différents milieux physiologiques in vivo et in vitro. Enfin, je signale l'action possible, dans certains cas, de la chaleur sur un début d'évolution in vitro de la métacercaire.

\section{Bibliographie}

Ciurea (I.) - Contribution à l'étude morphologique et biologique de quelques Strigéidés des oiseaux ichthyophages de la faune de Roumanie. Arch. Roumaines Pathol. exper. et microbiol., III, 1930, p. 277.

Dubors (G.). - Monographie des Strigeida (Trematoda). Mém. Soc. Neuchaleloise Sc. Nat., VI, 1937.

Scheuring (L.) et Evensвuch (E.). - Beiträge zur Entwicklungsgeschichte von Strigea (Holostomum) cornu Rud. Zool. Anz., LXVI, 1926, p. 41.

Institut d'Hygiène et de Bactériologie, Strasbourg. 